\title{
New technologies and architectures for laser systems: revolutionary beam control (Keynote Address)
}

Paul McManamon, William Thompson

Paul F. McManamon, William E. Thompson, "New technologies and architectures for laser systems: revolutionary beam control (Keynote Address)," Proc. SPIE 5413, Laser Systems Technology II, (8 September 2004); doi: $10.1117 / 12.548746$

SPIE. Event: Defense and Security, 2004, Orlando, Florida, United States 


\title{
New Technologies and Architectures for Laser Systems
}

\author{
- Revolutionary Beam Control -
}

Paul F. McManamon, Air Force Research Laboratory, 2241 Avionics Circle, building 620, Wright-

Patterson AFB, O 45433, 937-255-4039 ext 4024, paul.mcmanamon@wpafb.af.mil, and William

Thompson, Air Force Research Laboratory, Kirtland AFB, NM, 3550 Aberdeen Ave SE, Kirtland

AFB, NM 87117-5776, 505-846-2251, william.thompson@kirtland.af.mil

\begin{abstract}
This paper discusses revolutionary laser system architecture capable of dramatically reducing the complexity of laser systems while increasing capability. The architecture includes 3 major subsystems. The first is a phased array of laser sources. The second provides wavefront control and electronic beam steering. The third is sub-aperture receiver technology. Combining these three technologies into a new laser systems architecture results in a system that has graceful degradation, can steer to as wide an angle as individual optical phased array sub-apertures can steer, and can be scaled to high power and large apertures through phasing of a number of sub-apertures.
\end{abstract}

Keywords: Lasers, Fiber Lasers, Arrays, Optical Phased Arrays, Spatial light modulators, Sub-aperture receivers,

Sparse apertures

\section{INTRODUCTION}

Laser systems can be used for a wide range of applications, such as communications, sensing, and eventually weapons.

All applications can be significantly simplified and enhanced using the architecture discussed here. Simplification should result in a much lower lifetime cost for these laser systems, and therefore more widespread application. Large aperture laser systems will be dramatically lighter since the weight of the beam steering optics will only scale with area rather then to approximately the 2.7 power, as is the case with conventional systems. Traditional beam steering systems must become much thicker as the aperture becomes larger while a phased array of phased array non-mechanical system retains approximately the same thickness as total diameter increases. High-energy laser systems will of course have the largest gain in simplification in largest reduction in weight since they are the application requiring the largest aperture. In traditional HEL systems a high-bandwidth dynamic alignment system, coupled with sophisticated vibration isolation, is required to deal with jitter of the beam produced by the laser device and with the flexibility and vibration of the platform. 
A high-bandwidth, local-loop adaptive optics subsystem is required to deal with less-than-perfect beam quality produced by the laser device and with optical distortions due to heating of optical components by the laser beam. A separate target illuminator laser is required to meet signal-to-noise requirements for target tracking at high bandwidth, particularly to reject the jitter component of optical distortions introduced by atmospheric turbulence along the propagation path. A high-bandwidth, target-loop adaptive optics subsystem is required to deal with higher-order optical distortions introduced by atmospheric turbulence along the propagation path. An additional, separate beacon illuminator laser is required to meet signal-to-noise or point-ahead requirements for target-loop wave-front sensing at high bandwidth. Shared aperture tracking and target-loop wave-front sensing is required to eliminate the large non-common path errors that result from the use of a separate receive aperture.

To efficiently meet its design objectives, any laser system must address similar, if less stressing, requirements and constraints as outlined above. This paper addresses an alternative approach - one that takes advantage of the unique characteristics of optical phased arrays and that can easily handle the challenges described above in a single, mechanically simple, approach.

Phasing an array of fiber lasers has the potential to develop physically distributed, high efficiency, electrically pumped lasers that can be scaled to moderate or high power. This could replace the laser in laser systems. However, to make a dramatic difference in laser systems we also have to dramatically simplify the beam control system, and build an associated receiver. Just replacing the laser does not provide all the performance benefit associated with a PAPA architecture. The requirements to produce a coherent fiber laser array with both high power and good beam quality imply the capability to sense and control the phase of each element in the array and to expand and collimate the light from each element to produce a fully-filled, coherent, emitting aperture. These same capabilities can potentially contribute to some beam control functions, such as beam transport by fibers and piston phase control at the sub-aperture level for wave-front compensation and limited electronic beam steering.

Building on the inherent capabilities of phased array laser sources, it is conceptually possible to add additional technology 
elements in order to achieve the vision for an integrated phased array laser system. Specifically, the new technology elements needed are beam expansion so each fiber feeds a sub-aperture, sub-aperture tip and tilt wave-front control, and receive capabilities for target acquisition, wave-front sensing, target tracking, and laser beam pointing. Notionally, an integrated phased array laser system concept would be based on a phased array of diode-pumped fiber lasers, with each fiber feeding a crossed set of writable grating phased arrays. Figure 1 sows an implementation using multiple receive FPAs. Figure 2 shows an implementation using a single FPA and interfering the low resolution images from each subaperture.

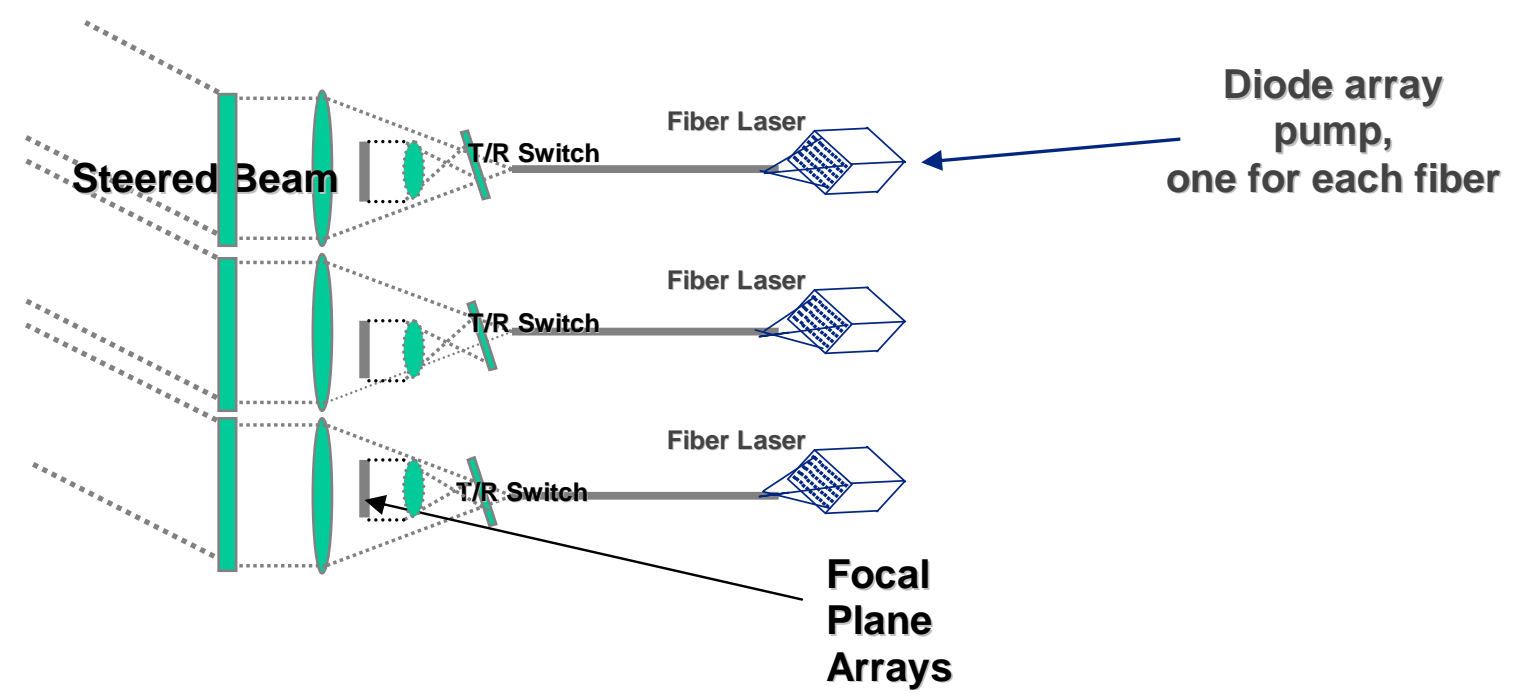

Figure 1: Array of Phased Array, PAPA, Concept Using Multiple FPAs 


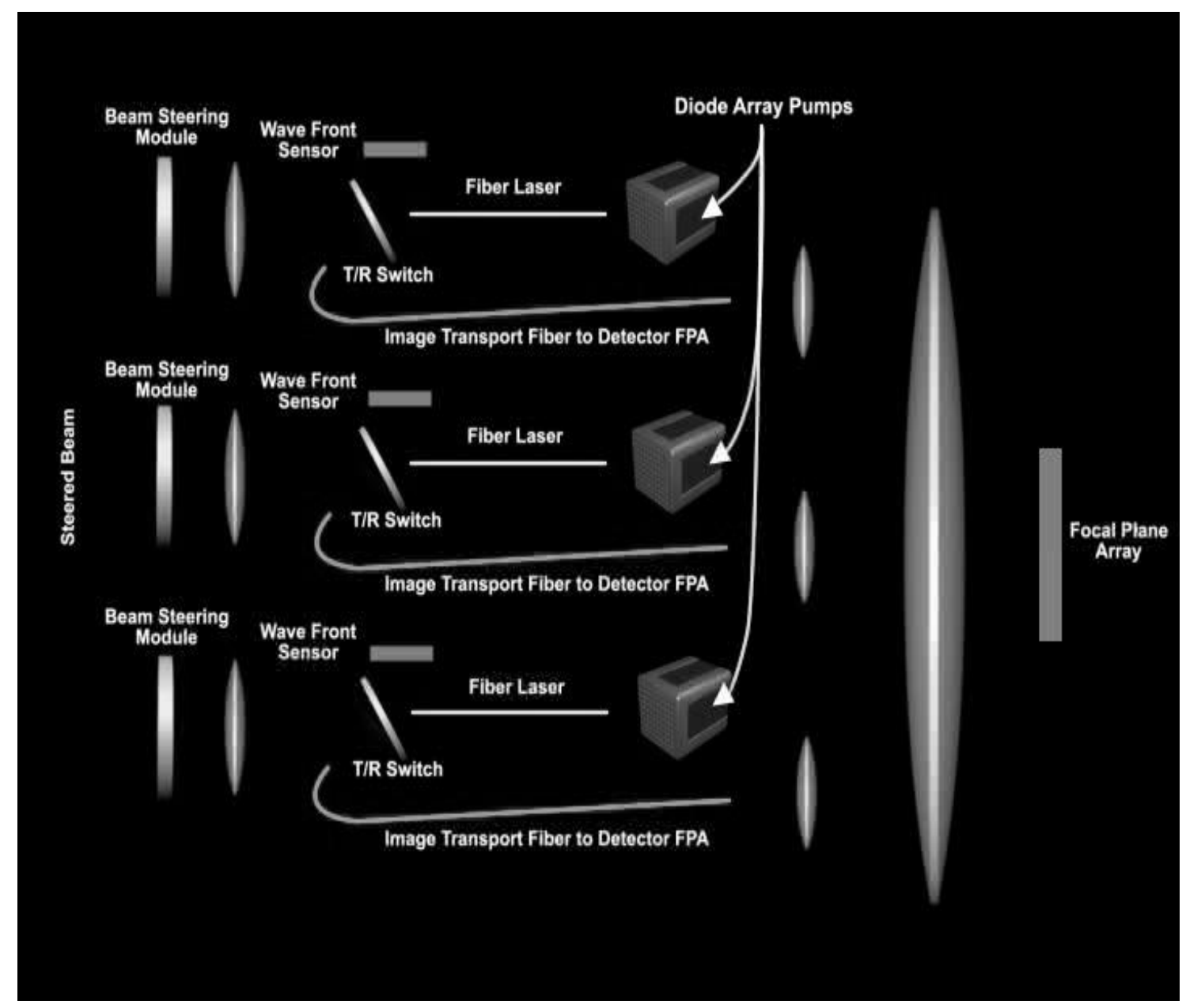

Figure 2 PAPA Concept Using a Single FPA

The fiber laser geometry couples naturally with phased array beam control, allowing the expansion to large phased-array aperture diameters without the use of complex beam train optics. Phase patterns will be able to be imposed on each subaperture, and in addition piston phase changes could be imposed from sub-aperture to sub-aperture. The ability to incorporate electronic wavefront control at the sub-aperture level would provide the capability for large, random angle electronic beam steering from the phased array aperture. Piston phase shifts from one sub-aperture to the next may allow true time delay phased arrays. Since this is a phased array implementation it would no longer be necessary, when going from angle one to angle two, to pass through all the angles in between. It would also be possible to electronically adjust focus and to electronically generate multiple beams in various patterns.

As discussed above, phased array laser system concepts represent an entirely different approach to the design of a laser 
system. As you add fibers \& sub-apertures the laser power increases and the aperture size increases. This is a scaleable concept. You might have a 100-watt PAPA system consisting of 25 four-watt fibers each fed into a $2 \mathrm{~cm}$ x $2 \mathrm{~cm}$ aperture. This results in a $10 \mathrm{~cm} \times 10 \mathrm{~cm}$ aperture of 100 watts. You also could have 2500-kilowatt fiber lasers, each feeding a 20 $\mathrm{cm}$ x $20 \mathrm{~cm}$ aperture. This yields a 2.5 Megawatt laser system with a 10 meter x 10-meter aperture. In concept neither of these systems has a complex beam control system.

If the attractive features of this concept can be realized in a practical and scalable system, it would have dramatic payoff across an exceptional range of laser system designs. The development of a phased array laser system conceptual design based on this vision offers significant challenges. Implementation approaches which best preserve the attractive features of the concept will require new technology approaches. Figure 3 shows why this is such an ideal approach to optical phased arrays. PAPA allows independent optimization of sub-aperture size, and beam steering.

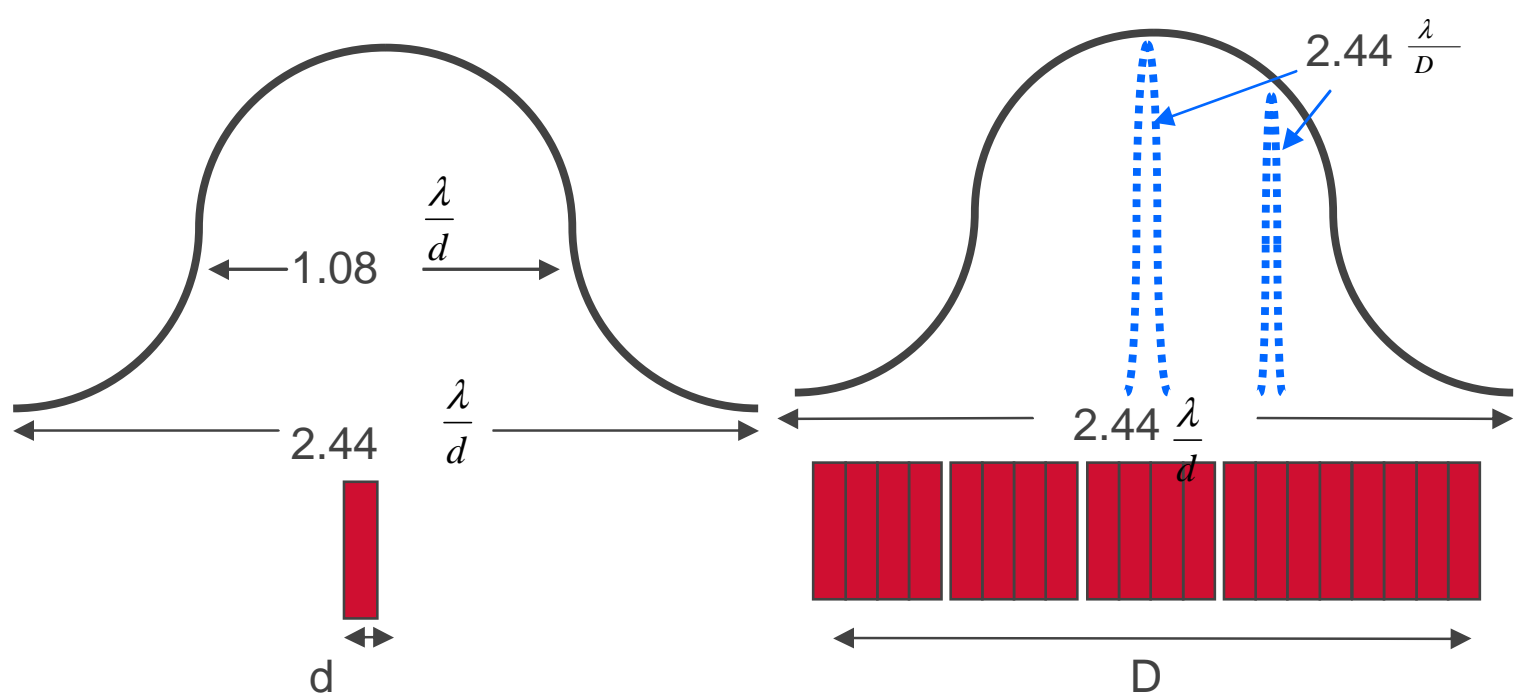

Figure 3: Beam With of an smallest addressable element in a sub-aperture sets the field of regard for the array beam steering

Technology development efforts are currently focused on individual optical phased array beam steerers. These devices are also sometimes called spatial light modulators. When applied at the sub-aperture level, this same technology may be applicable to the wavefront control and beam steering requirements for a phased array laser system.

A means to receive and process return signals from the target is necessary for target acquisition, tracking, aim point 
designation/control, and atmospheric compensation. While it is possible to use a separate, conventional aperture and sensor system to perform these functions, this extra aperture and beam train adds to the complexity (cost, weight,

integration issues) of the overall laser system. It is thus highly desirable to develop techniques for collecting and sensing the return signals in a shared aperture as a function of the phased array itself. One of the significant issues is whether or not we require resolution beyond the diffraction limit of the sub-apertures. For some applications, like lasercom, it is probably okay to remain with the resolution of a sub-aperture. In that case receive sub-apertures would only need to have signals aligned well enough to prevent loss of communications bandwidth. For sensing or for laser weapon applications however it is necessary to obtain resolution beyond the diffraction limit of a single sub-aperture. To obtain the resolution with the array of sub-apertures it will be necessary to interfere the low resolution images to obtain the high resolution array based image. This requires knowledge of phase and amplitude of each sub-aperture image.

For laser communications or laser radar application $1.5 \mu \mathrm{m}$ is an ideal wavelength. This wavelength has the advantage that much of the fiber communications technology can be used. Lasers are not yet very powerful at these wavelengths, but neither laser communications nor laser radar require high power. At most we would push for hundreds of watts to have a robust lasercom or laser sensing system. These levels would require an array of fibers. For a laser weapon application it is likely $1.06 \mu \mathrm{m}$ would be a preferred wavelength both because of available laser power and because of the improved diffraction limit at a shorter wavelength. Of course there will be additional atmospheric scattering at the shorter wavelength.

\section{OPTICAL PHASED ARRAY TECHNOLOGY}

Optical phased array function is well described in reference 1. ${ }^{\mathrm{i}}$ It can be used in each sub-aperture. As described in reference 1 increasing the optical path delay across the aperture can provide beam steering. For a single wavelength, one can subtract $2 \pi$ phase shift, and repeat the increase in phase shift. The phase ramp across the aperture subtracts $2 \pi$ every time the optical path delay becomes larger than $2 \pi$. This can be accomplished with a set of step phase shifters, and we can use crossed AZ / EL writable gratings, as shown in figure 4 . Figure 5 shows the 
phase ramp that can be placed across an aperture. One approach to generating the phase ramp is liquid crystals.

Figure 6 shows a liquid crystal cell with voltage across it to change the optical path delay.

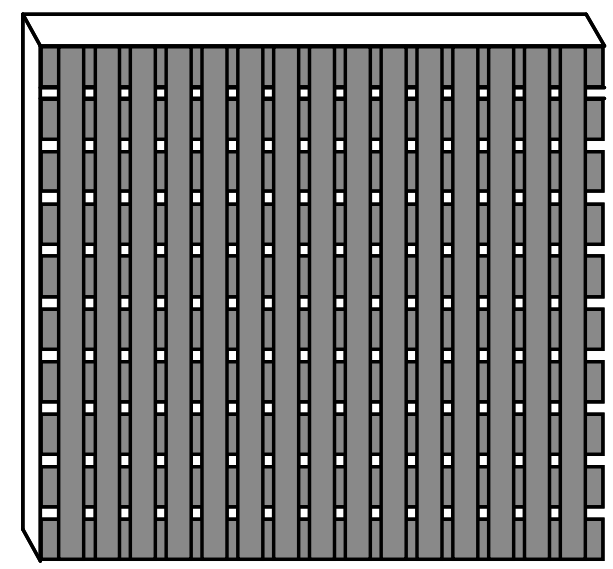

Figure 4 Crossed AZ / EL writable grating optical phased arrays

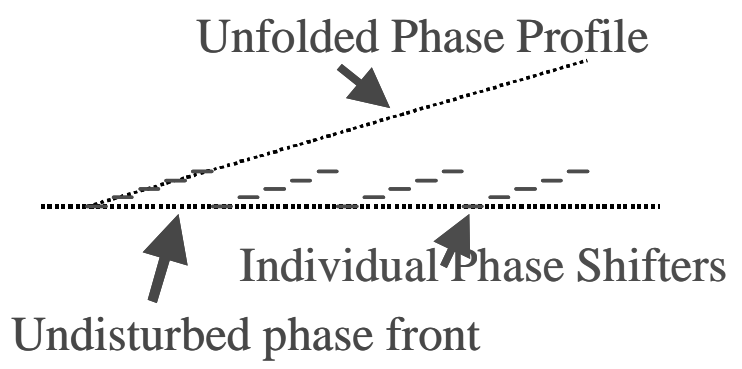

Figure 5 Phased Array phase Ramp with re-sets

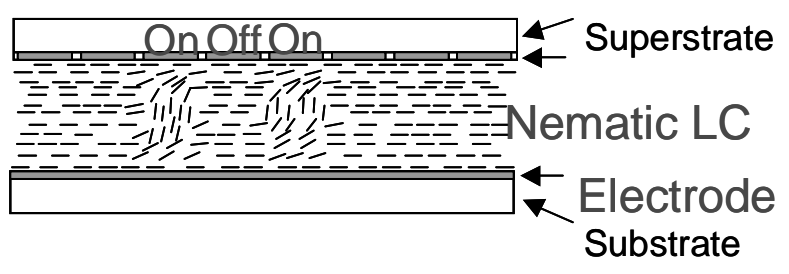

Figure 6 A Liquid Crystal Cell Cross section

When voltage is placed across the cell the optical path difference for a given polarization changes. This is a method of generating phase delays, up to a certain amount. The cell needs to be thicker to generate larger optical path delays, and 
therefore larger phase delays. As the cell becomes thicker the speed of the switch decreases as the square of the cell thickness. Therefore it makes sense to limit the required phase delay, and usually a modulo $2 \pi$ phase ramp approach is used. Figure 7 shows the graph of voltage vs. phase delay for a liquid crystal cell.

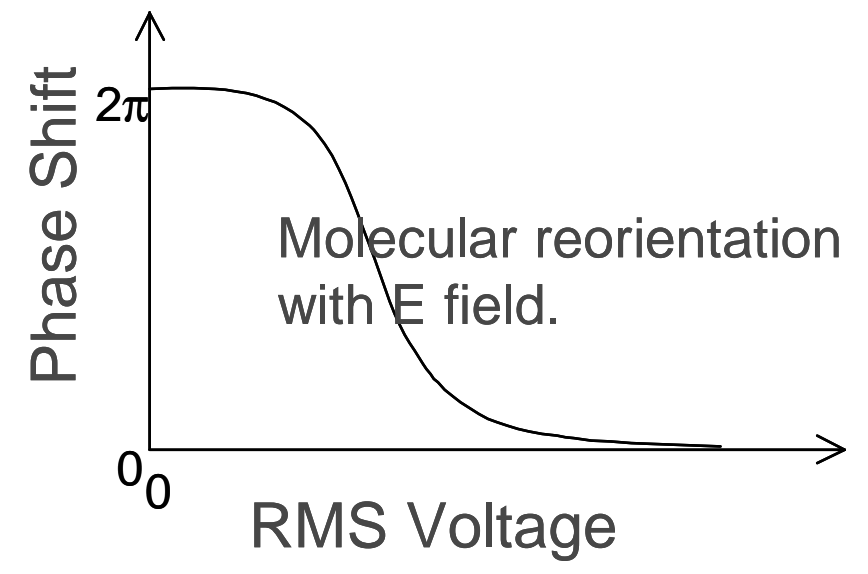

Figure 7 Phase Shift vs., voltage for a liquid crystal cell

To deflect a beam at large angles requires control of small phase retarding apertures. The rule of thumb used for phased array radar is that each phase retarding aperture should be one half wavelength center to center. If this is done, then it is possible to steer to 45 degrees deflection with approximately a cosine loss in steering efficiency. Unfortunately even if we can fabricate half wavelength center-to-center spacing electrodes it will be difficult to actually address the beam steerer at this accuracy. It is not a major difficulty while proceeding up the phase ramp, for example, going from a 30-degree phase shift to a 60-degree phase shift. At the reset however we need to go from 360 phase shift to 0 degree phase shift. Fringing fields will be a problem for accurate addressing during reset. If we take a 0.2 bi-refringence liquid crystal material, the cell must be 5 wavelengths thick for a transmissive cell, or 2.5 wavelengths thick for a reflective cell. Traditionally fringing fields are about as wide as the cell is thick. This means for 0.2 birefringence our best addressing is about 2.5 wavelengths in reflective mode or 5 wavelengths in transmissive mode. This is a factor of five to ten away from the ideal phased array spacing. If we can use advanced liquid crystal with a 0.4 bi-refringence then we will be a factor of 2.5 to 5 away from ideal center-to-center spacing. It may be possible to push the rule of thumb concerning fringing fields. If we can have fringing fields, which are not as wide as the cell is thick, we can get closer to good wide angle steering. The 
liquid crystal's coherence length may also limit the smallest effective center-to-center spacing. This is an alternate potential limiting mechanism for fine addressing. Liquid crystal cells close each other do not readily change polarization.

For a straight -forward phased array the maximum beam steering angle is:

$$
\theta \approx \frac{\lambda}{3 d}
$$

where the factor of 3 is a judgment factor depending on the efficiency threshold used. Using Eq. 1 we see the maximum deflection angle at this level of efficiency is plus or minus 4 degrees for .2 birefringence in a transmissive mode, and plus or minus 7.5 degrees for .2 birefringence in a reflective mode - so long as liquid crystal spatial coherence does not limit the addressing. For .4 birefringence we could double these angles.

Because of the limitation currently associate with maximum steering angle other approaches are being implemented. These include holographic glass, ${ }^{\mathrm{ii}}$ lenslet arrays ${ }^{\mathrm{iii}}$, multiple prisms, ${ }^{\mathrm{iv}}$ and other approaches. Figure 8 shows a couple wide angle beam steering approaches, one being pursued by Raytheon and one by Rockwell. These approaches are used to expand the field of regard of angular steering provided for optical phased array beam steering so we can address a full field of regard of $>90$ degrees. Crossed beam steering elements are required to steer in both azimuth and elevation. This combination of approaches again emphasizes the engineering benefit PAPA provided by separating the nonmechanical beam steering from sub-aperture size. 


\section{Wide Angle Beam Steering Approaches}

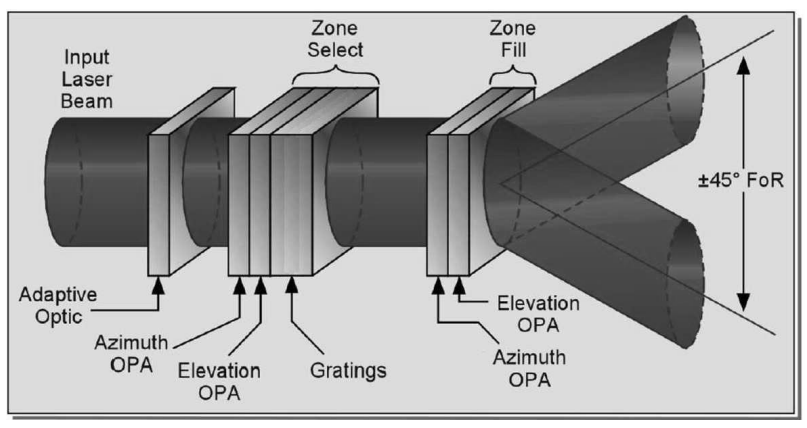

Raytheon Approach

- Liquid crystal zone select

- Holographic glass steering to large angles

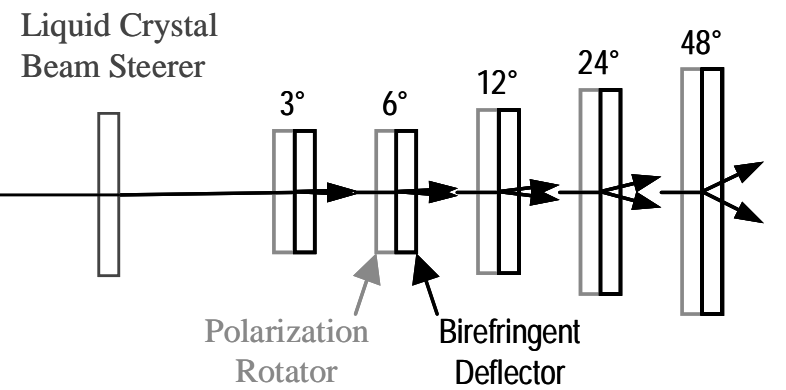

Rockwell Approach

- Liquid Crystal Fine angle

- Bi-refringent binary prisms for

large angles

Figure 8: Two Wide Angle Beam Steering Approaches

\section{PHASED ARRAY TRANSMITTERS}

A robust, integrated approach must be developed to ensure phase coherence and matching between all elements of the fiber laser array. A fiber array laser is shown in figure 9. As can be seen it starts with a single laser, and expands to multiple arrays of lasers.

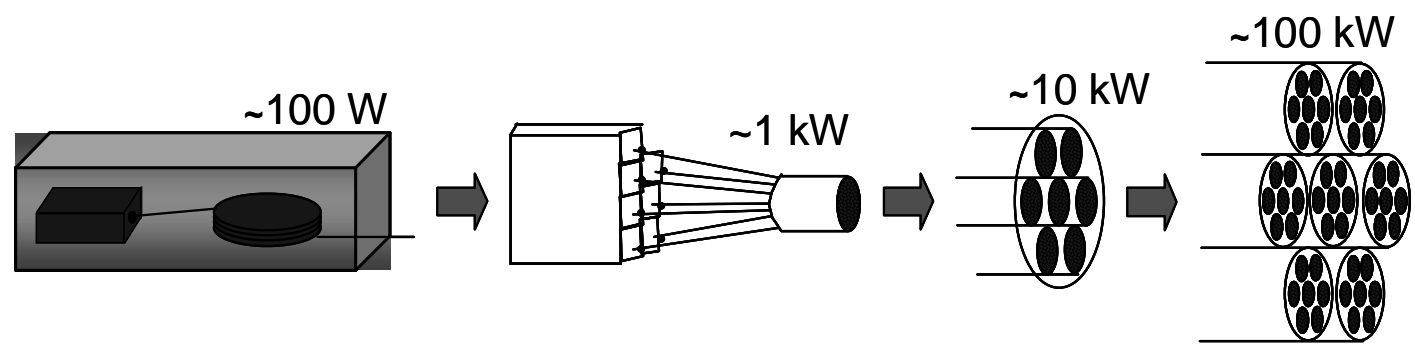

Figure 9 Phased Fiber Array Laser Architecture 
The most promising current candidate for ensuring phase coherence among array elements is the master oscillator-power amplifier concept, but the scalability of this concept must be evaluated and demonstrated, both in terms of the number of phased array elements and the laser power from each element. The traditional approach to ensure phase matching at a given plane involves the use of bulk beam splitters and the use of interferometer techniques to interrogate the phase of each element at that point. This information is then used to adjust each element's phase to achieve phase matching. This approach, while feasible, is complex to implement in a real system and may make such attractive features as conformal apertures impossible. Work is needed to investigate and develop new, integrated approaches based on fiber optic sensing to interrogate the phase of each element. This work may leverage current true time-delay fiber based optical phased array development work both for microwave radar and for optical phased array development.

New techniques will be needed to expand each beam from the core of each fiber laser ( $10 \mu \mathrm{m}$ in diameter) to achieve a high fill factor at the final projecting phased array aperture in order to achieve reasonable beam quality. Optical phased array sub-aperture sizes might be anywhere from $1 \mathrm{~cm}$ x $1 \mathrm{~cm}$ up to $20 \mathrm{~cm}$ x $20 \mathrm{~cm}$. Again, however, techniques that can be integrated with fiber lasers and concepts such as conformal apertures will have the most pay-off. By having each fiber feed a phased array sub-aperture we can keep down the number of required fibers to be phased, and we can use the individual phased array sub-apertures to steer over wide angles.

\section{PHASED ARRAY RECEIVERS}

Options to obtain high resolution images are shown in Figure 10. We can either use a single focal plane array and interfere 


\section{Sub-Aperture Receive Approaches \\ ** Most Challenging Technical Issue **}

\section{Fiber Image Transport}

- Can transmit sub-apertures images in multimode fiber or a fiber bundle

- Multimode fiber techniques have been demonstrated over short distances \& with small images (e.g. 9 x 9)

- Fiber bundle techniques are under development

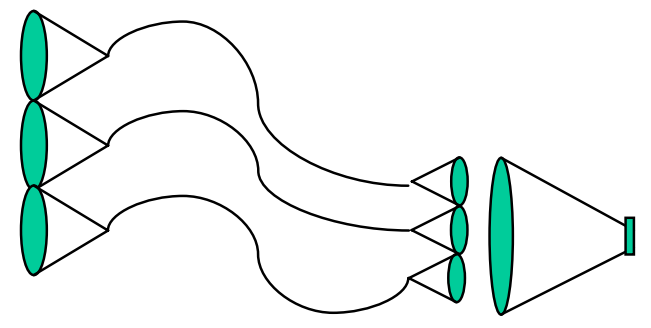

\section{Digital Image Transport}

- Images digitized at each sub-aperture FPA

- Need phase \& amplitude of images

- May get away with a single phase sample per image, or may need to sample full sub-aperture

- Great flexibility - parts of PAPA array can image various scenes

- Sub-aperture phase measurement, cost, \& computational requirements are challenges

FPA at Each Sub-Aperture 田田田田回回田

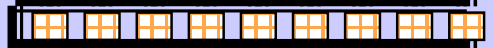

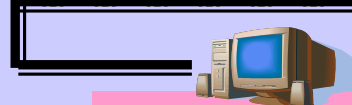

Preferred Approach

Fig. 10: Sub-Aperture Receive Options

sub-aperture images, or we can use a focal plane array at each sub-aperture and can measure or estimate phase at each subaperture. Using a single focal plane array is essentially coherent beam combining in reverse, in which the return pulse is transmitted over a fiber or fiber bundle to a focal plane array. We have the added complexities that phase must be preserved and each return fiber or fiber bundle must illuminate the focal plane array from the same direction it would have come from if the sub-aperture was part of a monolithic receiver aperture. In addition each fiber, or coherent fiber bundle, will have to be capable of transmitting a low resolution image from the sub-aperture receive element to the FPA so that these low resolution images can interfere at the FPA to produce the high resolution image. Figure 11 shows some options 


\section{Fiber Image Transport- Single multi-mode fiber}
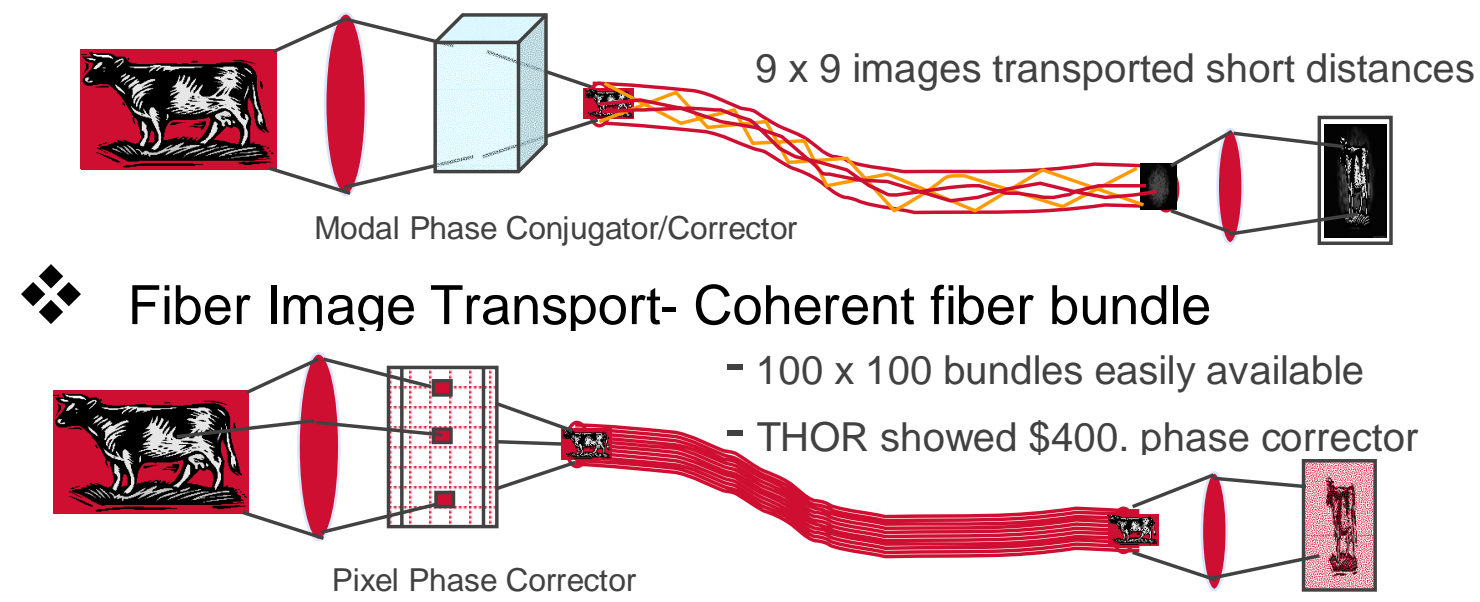

\section{* Combining Multiple Sub-apertures}

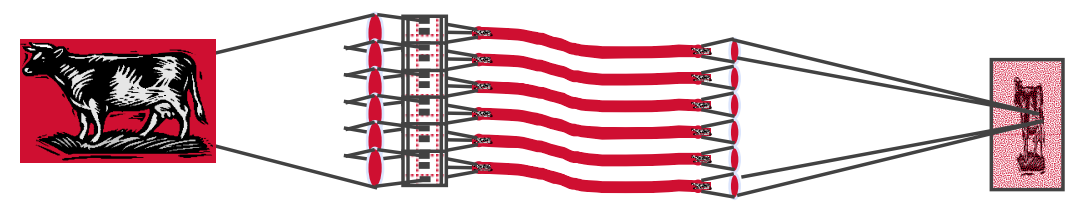

Fig: 11: Methods of sub-image fiber transport

for transmitting sub-aperture images through fibers. As can be seen either we are relegated to only transferring very low pixel count imagers or we must use a fiber bundle and probably also use a 2D spatial light modulator to compensate for fiber length variations. This is likely to be a complex approach, requiring complex alignment procedures. Fiber position in illuminating the focal plane array will be critical along with maintenance of phase. Each fiber or fiber bundle will have to be capable of accurately transmitting an image consistent with the diffraction limit of an individual PAPA sub-aperture. These sub-aperture images will interfere at the focal plane to create a high-resolution image consistent with the full set of sub-apertures. This is diagramed schematically in Fig 2. If we have a separate detector plane array at each sub-aperture it is preferred that we put the detector array in the pupil plane rather then in the image plane. This is a form of pupil plane imaging, and the high resolution image will be formed in a computer. Of course we will measure the amplitude at each detector array. Significant issues for investigation will be whether we measure phase, or not, and how many phase measurements do we make. For some images it will certainly be possible to not measure phase at all, but to construct a high resolution image. Image formation time will be an issue. Alternately we could make a single phase measurement 
within each sub-aperture, and assume that phase within a sub-aperture can be well estimated. Yet again, phase could be measured at each detector element. Heterodyne phase measure schemes should be easier with long coherence length laser light. Some techniques for forming the high resolution image will favor long coherence length while other will favor short coherence length.

\section{CONCLUSIONS}

The ability to build a laser system with a small number of components, no moving parts, and significant beam agility / flexibility will make future laser systems more attractive for all applications. Ongoing work in optical phased array beam steering and in arraying fiber lasers can be leveraged to make this happen. Additional work will be required in sub-aperture receiver techniques, and of course in the integration of these three technologies into a single new laser systems architecture. Laser systems using this architecture will be developed for medium to high power. The larger systems will take significant development, but will have the most payoff in terms of systems weight, reliability, and simplification.

\section{REFERENCES}

1) ${ }^{\text {i }}$ P. F. McManamon, T. A. Dorschner, D. C. Corkum, L. J. Friedman, D. S. Hobbs, M. K. O. Holz, S. Liberman, H. Nguyen, D. P. Resler, R. C. Sharp, and E. A. Watson, “Optical Phased Array Technology,” Proc. IEEE 84(2), 268-298 (1996).

2) ii Terry Dorschner, private communication

3) iii E. A. Watson, “Analysis of beam steering with decentered microlens arrays,” Opt. Eng. 32(11), 2665-2670 (1993).

4) ${ }^{\text {iv }}$ Phil Bos, private communication 